\title{
Aspectos legales del consentimiento informado en odontología.
}

\author{
Legal aspects of informed consent in dentistry.
}

\author{
Agustín Tiol-Carrillo*
}

\section{RESUMEN}

El consentimiento informado forma parte de los elementos que constituyen el expediente clínico. Por su importancia informativa sobre posibles riesgos y complicaciones inherentes al tratamiento a realizar, su elaboración resulta de vital importancia. Existen numerosas legislaciones y normatividades que regulan este notable documento; sin embargo, resulta alarmante que muchos profesionistas omiten su realización o lo elaboran erróneamente, poniéndolos en riesgo de sufrir problemas legales. Además, es una realidad que el consentimiento informado es un documento mal entendido por la mayoría de los profesionistas, pudiendo incurrir en numerosos errores por desconocimiento. El objetivo del presente artículo es informar al cirujano dentista sobre los verdaderos alcances del consentimiento informado con fundamento en la legislación vigente en México.

Palabras clave: Consentimiento informado, expediente clínico, odontología legal, responsabilidad profesional.

\section{ABSTRACT}

Informed consent is part of the elements that make up the clinical record. Due to its informative importance on possible risks and complications inherent to the treatment to be carried out, its preparation is of vital importance. There are numerous laws and regulations that regulate this remarkable document, however, it is alarming that many professionals omit its elaboration or wrongly elaborate it, putting them at risk of suffering legal problems. In addition, it is a reality that informed consent is a document misunderstood by most professionals, and may incur numerous errors due to ignorance. The aim of this article is to inform the dental surgeon about the true scope of informed consent based on current legislation in Mexico.

Keywords: Informed consent, medical records, legal dentistry, professional responsibility.

\section{INTRODUCCIÓN}

$\mathrm{D}_{\mathrm{s}}$ esde inicios de su formación profesional, el odontólogo es instruido sobre la importancia de la documentación médico legal que constituye el expediente clínico. Son numerosos los archivos que contiene el expediente clínico, y cada uno de ellos se considera un eslabón para la adecuada constitución de este documento de gran relevancia clínica y legal.

Su principal función es la de permitir al odontólogo tratante conocer los antecedentes heredofamiliares, patológicos y no patológicos de su paciente, información de gran relevancia para normar un plan de tratamiento y evitar eventos adversos durante la atención clínica, aunque su función legal radica en que en él se incluyen todos los elementos que hagan constar la buena práctica médica, a través del resguardo de los estudios y radiografías tomadas, las notas de evolución adecuadamente redactadas y el apropiado llenado del consentimiento informado $(\mathrm{Cl})$.

$\mathrm{El} \mathrm{Cl}$ es considerado como el acto más importante de la relación odontólogo-paciente, ya que, tras su llenado, se crean derechos y obligaciones para el odontólogo y para el paciente, ${ }^{1}$ y resulta inaudito que muchos profesionales de la salud omiten este importante documento previo a la

\footnotetext{
* Especialista en Estomatología Pediátrica. Máster en Odontología Legal y Forense. Profesor investigador en Universidad Autónoma Metropolitana-Xochimilco, CDMX.

Recibido: 02 de marzo de 2021. Aceptado: 18 de marzo de 2021.

Citar como: Tiol-Carrillo A. Aspectos legales del consentimiento informado en odontología. Rev ADM. 2021; 78 (2): 80-83. https://dx.doi. org/10.35366/99282
} 
intervención médica u odontológica a realizar, aun sabiendo las consecuencias negativas que pudieran presentarse.

Sin embargo, también es una realidad que el $\mathrm{Cl}$ es muchas veces malentendido por los propios profesionales de la salud, desconociendo su verdadera relevancia y pensando erróneamente que es un documento sin importancia clínica que sólo consume tiempo durante la consulta, o la creencia de que es la piedra angular para la protección del odontólogo en caso de negligencia o mala praxis, por lo que el objetivo del presente artículo es profundizar en el trasfondo ético y legal del $\mathrm{Cl}$.

\section{GENERALIDADES DEL CONSENTIMIENTO INFORMADO}

Son bien conocidos los actos inhumanos ejecutados durante la Segunda Guerra Mundial en los campos de concentración que vulneraban los derechos y la dignidad de todos sus internos. Además del genocidio dentro de los campos, la cruel experimentación con los reclusos de resultados generalmente letales era un evento cotidiano dentro de estos recintos. ${ }^{2}$ Por tanto, a raíz de este evento histórico tan desafortunado, se creó durante los Juicios de Núremberg el $\mathrm{Cl}$, siendo este un documento de carácter ético-normativo que se caracterizaba por dotar a cualquier individuo del derecho para poder participar voluntariamente en ensayos o investigaciones científicas, siempre y cuando exista un consentimiento por parte del individuo tras brindar una minuciosa información sobre el estudio o investigación a la que será sometido.

En México, el Cl es el acuerdo escrito mediante el cual el sujeto de investigación o, en su caso, su representante legal autoriza su participación en la investigación, con el pleno conocimiento de la naturaleza de los procedimientos y riesgos a los que se someterá, con la capacidad de libre elección y sin coacción alguna según lo especificado en el artículo 20 del Reglamento de la Ley General de Salud en Materia de Investigación para la Salud. ${ }^{3}$

En este mismo tenor, y de conformidad con el artículo 21 de dicho Reglamento, el $\mathrm{Cl}$ debe contener como mínimo los siguientes aspectos: justificación y objetivos de la investigación, los procedimientos que vayan a usarse y su propósito, las molestias o riesgos esperados, los beneficios a obtener, procedimientos alternativos que pudieran ser ventajosos para el sujeto, garantía de recibir respuesta a cualquier pregunta y aclaración a cualquier duda acerca de los procedimientos, riesgos y beneficios, la libertad de poder retirar su consentimiento en cualquier momento, la seguridad de que se mantendrá su confidencialidad. ${ }^{3}$
Como se puede ver en los párrafos anteriores, el Reglamento de la Ley General de Salud en Materia de Investigación para la Salud considera el Cl como un documento obligatorio exclusivamente en la investigación sanitaria, más no en procedimientos clínicos. No obstante, es la Norma Oficial Mexicana del Expediente Clínico en su numeral 4.2 la que exige su obligatoriedad antes de iniciar cualquier tratamiento médico, señalando que: la carta de consentimiento informado son los documentos escritos, signados por el paciente o su representante o familiar más cercano en vínculo, mediante el cual se acepta un procedimiento médico o quirúrgico con fines diagnósticos, terapéuticos, rehabilitatorios, paliativos o de investigación, una vez que se ha recibido la información de los riesgos y beneficios esperados para el paciente. ${ }^{4}$

\section{CONSIDERACIONES LEGALES DEL CONSENTIMIENTO INFORMADO}

Los Derechos Humanos son un conjunto de prerrogativas que se encuentran reconocidas en el Capítulo 1 de nuestra Constitución Política. Dicho capítulo menciona que estos derechos deben promoverse, respetarse, protegerse y garantizarse, por lo que el Estado está obligado a prevenir, investigar, sancionar y reparar las violaciones a los derechos humanos. ${ }^{5}$

Son numerosos los derechos humanos, dos de ellos de principal importancia para el presente tema, el Derecho al acceso a la información y el cual especifica que toda persona tiene derecho a ser informada y recibir información objetiva y oportuna; y el Derecho a la protección de datos personales, que menciona que todo individuo tiene derecho a que sus datos personales estén debidamente protegidos y tendrá en todo momento el derecho de acceder a ellos, así como rectificarlos o cancelarlos cuando estos se encuentren en posesión de otras personas. Por tanto, el $\mathrm{Cl}$ es en realidad un derecho humano, dada su naturaleza informativa veraz y oportuna, que además contiene información sensible de cada paciente.

Resulta interesante notar que el $\mathrm{Cl}$ es un documento que no se ha comprendido del todo en el actuar del odontólogo, ya que muchas veces existe un formato prediseñado que el paciente sólo debe firmar, ${ }^{6}$ sin que realmente dicho formato contenga las especificaciones, avisos y condiciones del tratamiento individualizado al que el paciente será sometido, acción que demuestra sin duda la falta de comprensión de este importante documento.

$\mathrm{El} \mathrm{Cl}$ debe contener específicos elementos para poder ser considerado como tal, sus componentes mín- 
imos necesarios se encuentran descritos en el numeral 10.1.1.8. de la NOM-004-SSA3-2012 Del expediente clínico (Tabla 1), sin que esto signifique que no se pueda detallar más profundamente la intervención a realizar, pues la esencia del $\mathrm{Cl}$ es brindar información clara, concisa y veraz acerca del procedimiento. Así pues, el $\mathrm{Cl}$ debe ser un documento individualizado, ya que las condiciones específicas de un paciente no son las mismas que las de otros individuos, a pesar de tratarse de la misma intervención.

Sin embargo, es importante observar que el numeral 10.1.2. de la misma Norma especifica aquéllos procedimientos que requieren obligatoriamente la elaboración de un $\mathrm{Cl}$, sólo algunos de ellos pueden ser ejecutados por el cirujano dentista: procedimientos que requieren anestesia general o regional, investigación en seres humanos, procedimientos diagnósticos y terapéuticos considerados como de alto riesgo, así como cualquier procedimiento que entrañe mutilación. ${ }^{4}$ Por lo tanto, los procedimientos que no se encuentren expresamente descritos en esta NOM pueden realizarse con o sin la existencia previa de un $\mathrm{Cl}$ por su poco potencial de riesgo, por ejemplo, procedimientos de odontología preventiva, consultas de revisión o seguimiento o intervenciones dentales que no requieran infiltración anestésica. La libre elección de su realización con el fin de informar al paciente sobre procedimientos no invasivos tiene más un trasfondo bioético que legal.

Además de tener todos sus componentes presentes, para que el $\mathrm{Cl}$ tenga valor debe brindar de la forma más sincera y completa posible la información acerca del padecimiento preexistente y los procedimientos a realizar, y ser aceptado por el paciente de forma libre y sin coer- ción alguna estando mentalmente apto para entender los alcances de lo escrito en él. ${ }^{6}$

En casos de urgencia, donde el paciente no se encuentre en condiciones de aceptar el procedimiento a realizar y no esté acompañado de ningún tutor o representante legal, el artículo 81 del Reglamento de la Ley General de Salud en Materia de Prestación de Servicios de Atención Médica menciona que está autorizado realizar el procedimiento terapéutico más conveniente para el paciente dejando constancia por escrito en el expediente clínico. ${ }^{7}$

Durante su actuar profesional, cualquier odontólogo, por más experimentado que sea, es susceptible a realizar equivocaciones que culminen en el daño a la salud de sus pacientes. Estas equivocaciones pueden ser el motivo de demandas contra el odontólogo, y durante un proceso legal la presentación de pruebas que puedan crear o extinguir responsabilidades al cirujano dentista es de gran relevancia, y sin duda un elemento determinante para la protección del profesional en estas circunstancias es el expediente clínico.

Existe la falsa creencia de que el $\mathrm{Cl}$ es un eximente de responsabilidad profesional en caso de negligencia y que, por tanto, durante un proceso judicial en contra del odontólogo por mala praxis, la preexistencia de un $\mathrm{Cl}$ disolverá por completo su grado de responsabilidad, y esto no es más que una falacia, ya que si bien $\mathrm{Cl}$ es un documento de carácter puramente informativo sobre los posibles riesgos inherentes al tratamiento a realizar, este jamás prevé eventos negligentes, imprudentes ni imperitos, actos que, de presentarse son ejecutados accidentalmente por el profesional de la salud, y tras su comisión el profesional de la salud deberá ser sancionado según sea la gravedad de su actuar, a pesar de contar o

Tabla 1: Componentes del Consentimiento Informado según la NOM-004-SSA3-2012 Del expediente clínico.

Componentes mínimos del consentimiento informado

- Nombre de la institución a la que pertenece el establecimiento o nombre, razón o denominación social del establecimiento

- Título del documento

- Lugar y fecha en que se emite

- Acto autorizado

- Señalamiento de los riesgos y beneficios esperados del acto médico autorizado

- Autorización del personal de salud para la atención, contingencias y urgencias derivadas del acto autorizado, atendiendo al principio de libertad prescriptiva

- Nombre completo y firma del paciente si su estado lo permite, o en caso contrario, su tutor o representante legal

- Nombre completo y firma del médico que proporciona la información y recaba el consentimiento para el acto específico que fue otorgado y datos del médico tratante

- Nombre completo y firma de dos testigos 
no con un Cl. Lo anterior encuentra su justificación en el artículo 1910 del Código Civil Federal que especifica que «el que obrando ilícitamente o contra las buenas costumbres cause un daño a otro está obligado a repararlo» ${ }^{8}$ y el artículo 228 del Código Penal Federal que indica que «todo profesionista será responsable de los delitos producidos en el ejercicio de su profesión y estará obligado a la reparación del daño por sus actos propios y por los de sus auxiliares». ${ }^{9}$

Por otro lado, durante el proceso judicial en contra de un odontólogo, en donde sus actos hayan sido correctamente ejecutados y la demanda se haya derivado de complicaciones inherentes al tratamiento o en su defecto, por negligencia del propio paciente, entonces el odontólogo podrá utilizar el $\mathrm{Cl}$ como elemento de prueba que le permita demostrar su inocencia, tomando en cuenta que el artículo 1910 anteriormente mencionado exige la reparación del daño a quien sea responsable, excepto si se demuestra que el daño se produjo por negligencia inexcusable de la víctima. ${ }^{9}$

\section{CONCLUSIONES}

La correcta elaboración de la documentación médica es de vital importancia para el conocimiento del estado de salud del paciente y en la prevención de problemas legales; sin embargo, de todos los elementos que constituyen al expediente clínico, el Cl es de los más importantes por ser considerado como derecho humano y por brindar al paciente toda la información necesaria para la comprensión de los alcances de un determinado tratamiento médico. No obstante, de acuerdo con nuestra legislación, el
$\mathrm{Cl}$ debe realizarse exclusivamente en determinadas intervenciones y no cualquier procedimiento exige su elaboración. Además, durante procesos judiciales el $\mathrm{Cl}$ podrá ser de gran ayuda para la defensa del odontólogo, excepto cuando el motivo de la demanda sea por actos negligentes o por mala práctica médica, en donde el profesional responsable deberá reparar los daños correspondientes.

\section{REFERENCIAS}

1. Vázquez GA, Ramírez BE, Vázquez RJ, Cota GF, Gutiérrez MJA. Consentimiento informado ¿Requisito legal o ético? Cir Gen. 2017; 39 (3): 175-182.

2. Riquelme $U$, Horacio LA. Medicina bajo el nazismo: una aproximación histórico-cultural. Segunda parte. Medicina UPB. $2004 ; 23$ (1): 25-47.

3. Reglamento de la Ley General de Salud en Materia de Investigación para la Salud. Cámara de Diputados. 2014.

4. Norma Oficial Mexicana 004 Del expediente clínico. Diario Oficial de la Federación. 2012.

5. Constitución Política de los Estados Unidos Mexicanos. Cámara de Diputados. 2021.

6. Navarro-Reynoso FP, Argüelles-Mier M, Cicero-Sabido R. Derechos humanos y consentimiento informado. Cir Ciruj. 2004; 72: 239-245.

7. Reglamento de la Ley General de Salud en Materia de Prestación de Servicios de Atención Médica. Cámara de Diputados. 2018.

8. Código Civil Federal. Cámara de Diputados. 2021.

9. Código Penal Federal. Cámara de Diputados. 2021.

$$
\begin{aligned}
& \text { Correspondencia: } \\
& \text { Agustín Tiol-Carrillo } \\
& \text { E-mail: agustintiolcarrillo@gmail.com }
\end{aligned}
$$

Conflicto de intereses: El autor declara no tener ningún conflicto de intereses.

Aspectos éticos: Ninguno.

Financiamiento: Ninguno. 